\title{
Rede Combinatória de Modelos Não \\ Lineares - Parte II: Aplicação em Computação Aritmética *
}

\author{
Luan Pascoal da Costa Andrade* Márcia L. C. Peixoto** \\ Samir Angelo Milani Martins* \\ * GCOM - Grupo de Controle e Modelagem \\ Universidade Federal de São João del Rei Praça Frei Orlando, 170 \\ Centro. São João del Rei, MG, Brasil. (e-mail: \\ luan_pascoal13@hotmail.com matins@ufsj.edu.br) \\ ** Programa de Pós-Graduação em Engenharia Elétrica \\ Universidade Federal de Minas Gerais, Belo Horizonte, MG, Brasil. \\ (e-mail:marciapeixoto93@hotmail.com)
}

\begin{abstract}
:
This work is the second part of a series of two articles and applies a proposed method of parallel combination of models in the context of arithmetic computation. The combination is performed from known submodels obtained through an interval system identification procedure that uses interval arithmetic in the least squares estimator to find a set of models that best fits the data. The interpolated model was compared with a model obtained from a classic identification procedure and with the average model of the interval identification procedure obtaining a decrease of about $11 \%$ in the RMSE index. The model was also compared to the submodels used to obtain it, reaching in this case a decrease of about $79 \%$ in the RMSE and $22 \%$ in the energy of the autocorrelation of the waste vector.

Resumo: Este trabalho é a segunda parte de uma série de dois artigos e aplica um método proposto de combinação paralela de modelos no contexto da computação aritmética. A combinação é realizada a partir de submodelos conhecidos obtidos por meio de um procedimento de identificação intervalar de sistemas que usa aritmética intervalar no estimador de mínimos quadrados para encontrar um conjunto de modelos que melhor se ajusta aos dados. O modelo interpolado foi comparado com um modelo obtido a partir de um procedimento clássico de identificação e com o modelo médio do procedimento de identificação intervalar obtendo uma diminuição de cerca de $11 \%$ no índice RMSE. O modelo também foi comparado com os submodelos utilizados em sua obtenção obtendo neste caso uma diminuição de cerca de $79 \%$ no RMSE e de $22 \%$ na energia da autocorrelação do vetor de resíduos.
\end{abstract}

Keywords:

Systems Identification, Arithmetic Computation, Combinatorial Network of Models, Identification by Interval Arithmetic.

Palavras-chaves: Identificação de Sistemas, Computação Aritmética, Rede Combinatória de Modelos, Identificação por Aritmética Intervalar.

\section{INTRODUÇÃO}

A identificação de sistemas é uma área de estudo que lida com a obtenção de modelos matemáticos para sistemas de diversas naturezas como sistemas econômicos, processos químicos, sistemas mecânicos, elétricos entre outros (Ok et al., 2018; Chevtchenko et al., 2018; Zhao et al., 2018; Milovanović et al., 2017; Boda et al., 2016; Aguirre et al., 2011). Modelos matemáticos podem ser vistos como aproximações por meio de equações de um sistema real (Martins et al., 2013). Vários tipos de representações podem ser utilizadas para se modelar um sistema, como é o caso

* O presente trabalho foi realizado com apoio da Coordenação de Aperfeiçoamento de Pessoal de Nível Superior - Brasil (CAPES) Código de Financiamento 001. das Redes Neurais (Narendra and Parthasarathy, 1990), (Chen and Billings, 1992), modelos NARMAX (do inglês Nonlinear AutoRegressive Moving Average with eXogenous input) (Chen and Billings, 1989) nos quais podem ser citadas as representações polinomiais (Aguirre et al., 1998).

$\mathrm{Na}$ identificação de sistemas é indispensável o uso de simulações computacionais, sendo geralmente um conjunto de operações matemáticas executado de forma recursiva. Embora exista um grande desenvolvimento na simulação computacional, muitos dos resultados obtidos pelos computadores não são exatos (Gao et al., 2018). Esse fato ocorre devido à representação finita dos números reais na máquina e ao realizar operações matemáticas dentro do computador erros de arredondamento se acumulam e 
se propagam a cada operação (Nepomuceno, 2014). Tal fenômeno pode ser observado em (Nepomuceno and Martins, 2016), que mostram que modelos NARMAX polinomiais matematicamente idênticos, com diferenças apenas do ponto de vista da representação em ponto flutuante podem resultar em respostas diferentes.

Nepomuceno et al. (2018) mostram que para o mesmo mapa logístico com as mesmas condições iniciais simulados apenas com diferenças de sintaxe (aplicando a propriedade associativa da multiplicação) as respostas real e simulada começam a divergir a partir da $80^{\mathrm{a}}$ iteração, apesar de Hammel et al. (1987) terem indicado um valor de $10^{7}$ iterações para a pseudo-órbita divergir da órbita real. Isso nos mostra uma influencia na quantidade de operações realizadas pelo computador e a propagação do erro numérico devido à representação em ponto flutuante.

A aritmética intervalar é uma técnica utilizada para levar em conta erros de arredondamento na computação numérica, bem como para avaliar os efeitos de erros de aproximações de entradas imprecisas. Nesta abordagem as grandezas são denotadas por um limite inferior e um limite superior, obtendo assim um intervalo no qual o valor real a ser representado está contido.

A aritmética intervalar é utilizada no contexto da identificação de sistemas em (Peixoto et al., 2017). O trabalho propõe a realização da estimação de parâmetros na identificação de modelos NARMAX polinomiais utilizando a aritmética intervalar. O procedimento proposto gera então não apenas um modelo, mas sim um conjunto de modelos no qual está contido o modelo que seria identificado por um procedimento padrão de identificação.

A abordagem proposta por Peixoto et al. (2017) apresenta um conjunto de modelos. Entretanto, muitas vezes é necessário um único modelo que represente o sistema em estudo. A partir disso, este trabalho que é a segunda parte de uma série de 2 artigos, aplica o método proposto em sua primeira parte Rede Combinatória de Modelos Não Lineares - Parte I: Método e Aplicação em Identificação Multiobjetivo de Sistemas, para combinar modelos pertencentes ao conjunto de modelos intervalares. Assim, é possível encontrar dentro do intervalo de modelos obtidos pela identificação intervalar um único modelo que melhor se ajuste a dados auxiliares.

O restante do artigo é organizado da seguinte forma. A Seção 2 traz os conceitos preliminares necessários no entendimento do trabalho. Na Seção 3 a metodologia é descrita e os resultados obtidos na implementação da mesma são apresentados na Seção 4. Por fim a Seção 5 apresenta as conclusões do artigo e perspectivas de trabalhos futuros.

\section{CONCEITOS PRELIMINARES}

Esta seção traz um breve referencial teórico necessário ao entendimento do trabalho.

\subsection{Modelos NARMAX}

Os modelos NARMAX (Nonlinear AutoRegressive Moving Average with eXogenous inputs) são representações matemáticas caracterizados por utilizar combinações lineares e não lineares de suas entradas e saídas em instantes passados, os chamados regressores, para determinar a saída presente. Os modelos NARMAX polinomiais são representações autorregressivas compostas por polinômios das entradas e saídas passadas, e podem ser escritos como em (Leontaritis and Billings, 1985a,b) (1):

$$
\begin{aligned}
y_{k}= & F^{\ell}\left[y_{k-1}, \ldots, y_{k-n_{y}}, u_{k-1}, \ldots\right. \\
& \left.u_{k-n_{u}}, e_{k-1}, \ldots, e_{k-n_{e}}\right]+e_{k}
\end{aligned}
$$

em que $y_{k}, u_{k}$ e $e_{k}$ são a saída, a entrada e a incerteza (ou ruído) respectivamente, e $n_{y} n_{u}$ e $n_{e}$ os atrasos máximos correspondentes (Chen and Billings, 1989). $F^{\ell}[\cdot]$ é uma função polinomial dos regressores de entrada e saída (Aguirre and Billings, 1995).

\subsection{Critérios de Validação}

Raiz do Erro Quadrático Médio Normalizado O NRMSE ou Raiz do Erro Quadrático Médio Normalizado é um índice utilizado para avaliar o desempenho de modelos e pode ser calculado como em (2):

$$
\operatorname{NRMSE}=\frac{\sqrt{\sum_{k=1}^{N}\left(y_{k}-\hat{y}_{k}\right)^{2}}}{\sqrt{\sum_{k=1}^{N}\left(y_{k}-\bar{y}_{k}\right)^{2}}} .
$$

em que $\hat{y}_{k}$ é a saída do modelo, $y_{k}$ a saída real do sistema e $\overline{y_{k}}$ sua média.

Análise de Resíduos Na análise de resíduos são efetuados alguns testes com a finalidade de verificar se os resíduos, ou seja, os erros cometidos pelos modelos possuem características aleatórias ou não. É desejável que o resíduo possua as características de um ruído branco e que não seja correlacionado com a entrada. Alguns dos testes que podem ser executados são a correlação cruzada entre o vetor de resíduos e a entrada (3) e a auto correlação do vetor de resíduos (4) (Aguirre, 2015).

$$
\begin{aligned}
& r_{u \xi}(k)=\lim _{N \rightarrow \infty} \frac{1}{2 N+1} \sum_{i=-n}^{n} u(i) \xi(i+k) \\
& r_{\xi \xi}(k)=\lim _{N \rightarrow \infty} \frac{1}{2 N+1} \sum_{i=-n}^{n} \xi(i) \xi(i+k)
\end{aligned}
$$

em que $\xi$ é o vetor de resíduos, $u$ é o vetor de entrada, $k$ indica tempo discreto e $N$ a quantidade de amostras.

\subsection{Identificação de Sistemas com Aritmética Intervalar}

$\mathrm{Na}$ aritmética intervalar as grandezas não são representadas como um único número e sim como um intervalo $X$ possuindo um limite superior $\bar{X}$ e um limite inferior $\underline{X}$, sendo normalmente representado como $X=[\underline{X}, \bar{X}]$ (Moore et al., 2009).

A identificação de sistemas via aritmética intervalar utiliza na etapa de estimação dos parâmetros, a inserção de intervalos no cálculo do método de mínimos quadrados, com o intuito de manusear os erros numéricos, obtendo os mínimos quadrados intervalar. 
Os parâmetros obtidos para o modelo não são valores únicos e sim um intervalo de valores, sendo então identificado um intervalo de modelos que podem ser calculados como:

$$
\hat{\theta_{\mathbf{I}}}=\left[\Psi_{I}^{T} \Psi_{I}\right]^{-1} \Psi_{I}^{T} y,
$$

em que $\hat{\theta_{\mathbf{I}}}=[\underline{\hat{\theta}}, \overline{\hat{\theta}}]$, é o intervalo de coeficientes calculados $\Psi_{I_{i, j}}=\left[\Psi_{i, j}, \overline{\Psi_{i, j}}\right]$ é a matriz de regressores intervalar, em que $i$ e $j \overline{\text { são }}$ os índices da linha e da coluna da matriz $\Psi_{I}$, e $y$ é o vetor de dados.

Os modelos intervalares utilizados neste trabalho foram obtidos em (Peixoto et al., 2017). A estrutura foi selecionada a partir da Taxa de Redução de Erro juntamente com o Critério de Informação de Akaike. A estrutura selecionada pode ser vista em (6). Para a seleção dos parâmetros utilizou-se os Mínimos Quadrados Intervalares como em (5). Os parâmetros selecionados para o modelo podem ser vistos em (7).

$$
\begin{aligned}
\Psi= & {\left[\begin{array}{llll}
y(k-1) & y(k-2) & u(k-1) & u(k-1) y(k-1)
\end{array}\right.} \\
& u(k-2) u(k-1) y(k-2) \quad u(k-2) y(k-1) \\
& \left.u(k-2) y(k-2) \quad y(k-2)^{2}\right] .
\end{aligned}
$$

$$
\hat{\theta}=\left[\begin{array}{rr}
{[1,86983289867678 ;} & 1,87052216754597] \\
{[-0,87500271106274 ;} & -0,87433604737629] \\
{[0,00877520297552 ;} & 0,07522496598523] \\
{[-0,07754127207970 ;} & -0,07522496598523] \\
0,01156721443224 ; & 0,01159157820069] \\
{[0,06736048557893 ;} & 0,06969424138646] \\
{[-0,09177956625574 ;} & 0,08882860964415] \\
{[0,07814928825610 ;} & 0,08111662045620] \\
{[0,00273140158559 ;} & 0,00275676329139]
\end{array}\right] .
$$

Em que $\hat{\theta}$ são os intervalos de parâmetros mada o modelo identificado.

\section{METODOLOGIA}

Este trabalho propõe a aplicação da metodologia apresentada em sua primeira parte no contexto da identificação intervalar de sistemas. É realizada então a combinação paralela de submodelos obtidos por meio do procedimento de identificação intervalar descrito em (Peixoto et al., 2017), obtendo assim um único modelo que melhor se ajuste a dados auxiliares. O método é descrito na primeira parte do trabalho e será replicado aqui para fácil referência do leitor.

A Figura 1 mostra um diagrama da composição do modelo em que $u$ é a entrada do modelo, $y_{1}$ e $y_{2}$ são os modelos utilizados na composição e $\mu_{1}$ e $\mu_{2}$ seus respectivos pesos. $y^{*}$ é a resposta do modelo composto. Como submodelos $y_{1}$ e $y_{2}$ foram escolhidos o modelo do limite inferior e o modelo do limite superior do intervalo de modelos obtidos em (Peixoto et al., 2017) representados pelos parâmetros $\underline{\theta}_{n}$ e $\bar{\theta}_{n}$ de $(5)$.

Pela Figura 1 percebe-se que a composição do modelo $y^{*}$ trata-se de uma soma ponderada dos submodelos $y_{1}$ e $y_{2}$ pelos pesos $\mu_{1}$ e $\mu_{2}$ respectivamente. Para a aplicação da metodologia o problema deve estar bem condicionado

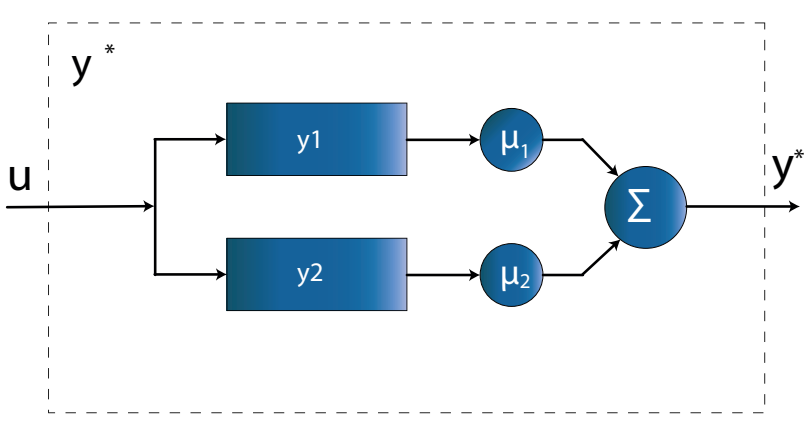

Figura 1. Rede Combinatória, sendo $y^{*}$ o modelo composto, $y_{1}$ e $y_{2}$ os submodelos utilizados e $w_{1}$ e $w_{2}$ seus respectivos pesos. $u$ é a entrada.

como na Figura 2, ou seja, a resposta real do sistema deve estar contida na região que compreende as respostas dos dois submodelos utilizados na combinação.

Variando os pesos entre 0 e 1 , assumindo $\mu_{1}+\mu_{2}=1$ a resposta $y^{*}$ do modelo composto exibido na Figura 1 se desloca na região compreendida pelas respostas dos modelos $y_{1}$ e $y_{2}$.

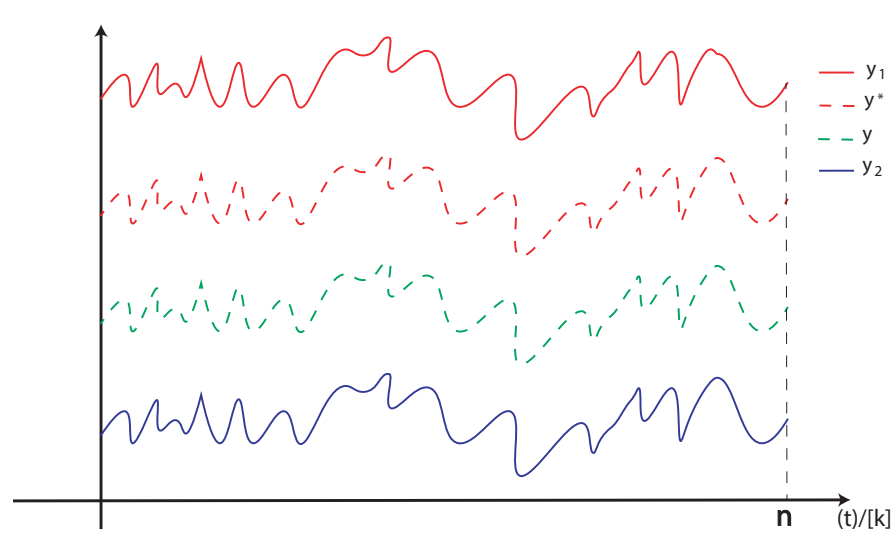

Figura 2. Saídas de modelos fictícios devido a entrada $u$. A resposta real do sistema deve estar compreendida entre a resposta dos modelos $y_{1}$ e $y_{2}$.

O modelo composto exibido pela Figura 1 pode ser definido como em (8). Assumindo $\mu_{2}=1-\mu_{1}$, o modelo pode ser representado ainda por $(9)$.

$$
\begin{gathered}
y^{*}=\mu_{1} y_{1}+\mu_{2} y_{2} . \\
y^{*}=\mu_{1} y_{1}+\left(1-\mu_{1}\right) y_{2} .
\end{gathered}
$$

Deseja-se que a saída do modelo composto seja a mais aproximada possível da saída real do sistema, ou seja $y^{*} \approx y$. Levando essa informação em (9) e abandonando o subscrito 1 em $\mu_{1}$ :

$$
y=\mu y_{1}+(1-\mu) y_{2}
$$

Não se conhece as funções representadas por $y, y_{1}$ e $y_{2}$ porém, uma vez que os modelos são estáveis, é possível calcular sua área. Integrando os dois lados da Equação (10):

$$
\int_{0}^{n} y d t=\int_{0}^{n} w y_{1}+(1-w) y_{2} d t
$$


Reagrupando os termos em (11) pode-se então encontrar uma estimativa do valor $\mu$ que faça com que $y^{*}$ seja aproximadamente $y$ :

$$
\mu=\frac{\int_{0}^{n} y(t) d t-\int_{0}^{n} y_{2}(t) d t}{\int_{0}^{n} y_{1}(t) d t-\int_{0}^{n} y_{2}(t) d t}
$$

Ou no domínio do tempo discreto:

$$
\mu=\frac{\sum_{k=0}^{n} y_{k}-\sum_{k=0}^{n} y_{2 k}}{\sum_{k=0}^{n} y_{1 k}-\sum_{k=0}^{n} y_{2 k}} .
$$

A abordagem foi testada através de um modelo identificado a partir de aritmética intervalar em (Peixoto et al., 2017). Foi utilizado o modelo de um sistema eletromecânico composto por duas maquinas $\mathrm{CC}$ acopladas sendo uma funcionando como motor e outra como gerador. O sistema foi excitado por um Sinal Binário Pseudo Aleatório (PRBS) e foi identificado utilizando a taxa de redução de erro (ERR) e o critério de informação de Akaike na seleção de estrutura e teve os parâmetros selecionados através de Mínimos Quadrados e Mínimos Quadrados Intervalar. A Estrutura do modelo utilizada pode ser vista em (6).

Em (Peixoto et al., 2017) é obtida uma família de modelos representada por um intervalo de parâmetros como indicado na Equação (5). Como submodelos $y_{1}$ e $y_{2}$ foram utilizados os modelos dos extremos deste intervalo, ou seja $\underline{\theta}_{n}$ e $\bar{\theta}_{n}$ certificando assim que o problema esteja condicionado como na Figura 2.

Os resultados obtidos para o modelo composto foram comparados com os resultados obtidos pelos submodelos utilizados em sua obtenção. O modelo composto foi comparado com mais dois modelos sendo um identificado por Mínimos Quadrados e o outro sendo o modelo do ponto médio do intervalo de modelos identificado pelo Mínimos Quadrados Intervalar aqui chamados de $y_{M M Q}$ e $y_{M M Q i}$ respectivamente.

\section{RESULTADOS}

A metodologia foi empregada utilizando o modelo do extremo superior da identificação intervalar como $y_{1} \mathrm{e}$ o modelo do extremo inferior como $y_{2}$. A resposta do modelo combinado $y^{*}$ em comparação com as respostas dos modelos $y_{1}$ e $y_{2}$ pode ser vista na Figura 6 , enquanto a comparação da resposta do modelo composto com o modelo identificado a partir de um procedimento padrão de identificação $y_{M M Q}$ pode ser visto na Figura 5 .

O modelo foi validado a partir do índice NRMSE e por meio da análise dos resíduos de predição livre. Nesta última calculou-se a autocorrelação do vetor de resíduos de predição livre $\xi$ bem como a correlação cruzada entre o vetor de resíduos $\xi$ e o vetor de entrada $u$ para o modelo combinado e para o modelo obtido por um procedimento padrão de identificação. O objetivo da análise é verificar se os resíduos possuem características de um ruído branco e se eles estão correlacionados com a entrada, ou seja, se há alguma sistematização do resíduo em relação a entrada. A Figura 3 mostra a função de autocorrelação para o modelo interpolado $y^{*}$ e para o modelo obtido por um procedimento clássico de identificação $y_{M M Q}$. A
Figura 4 mostra a função de correlação cruzada entre os resíduos do modelo interpolado e para o modelo obtido pelo procedimento padrão de identificação. Pode ser visto em ambas Figuras que o resíduo dos modelos obtidos possuem características aleatórias.

De uma forma qualitativa, a análise de resíduos apresentou resultados semelhantes para ambos os modelos. Para quantificar tal análise foram calculadas as energias das funções de autocorrelação e correlação cruzada. A Tabela I compara o índice NRMSE e a energia das funções de correlação entre o modelo composto $y^{*}$, os modelos $y_{1}$ e $y_{2}$ utilizados em sua obtenção, o modelo do ponto médio do intervalo de modelos $y_{M M Q i}$ e o modelo obtido por meio de um procedimento padrão de identificação $y_{M M Q}$.

Tabela I. Comparação entre os índices NRMSE e as energias das funções de correlação

\begin{tabular}{|l|l|l|l|}
\hline Modelo & NRMSE & $E_{r_{\xi \xi}}$ & $E_{r_{\xi u}}$ \\
\hline$y_{1}$ & 0,6930 & 17,56 & 1,69 \\
\hline$y_{2}$ & 0,5766 & 20,95 & 1,65 \\
\hline$y_{M M Q}$ & 0,1336 & 13,67 & 1,73 \\
\hline$y_{M M Q i}$ & 0,1336 & 13,67 & 1,73 \\
\hline$y^{*}$ & 0,1190 & 13,62 & 1,77 \\
\hline
\end{tabular}

Pela Tabela I percebe-se que o modelo obtido a partir da identificação clássica $y_{M M Q}$ e o modelo médio obtido por meio da identificação intervalar $y_{M M Q i}$ obtiveram o mesmo desempenho. Se comparado com estes, o modelo obtido por meio do procedimento proposto obteve uma diminuição de cerca de $11 \%$ para o índice NRMSE e uma diminuição de $0,36 \%$ na energia da autocorrelação do vetor de resíduos de predição livre, mostrando maior representatividade do sistema neste ponto de vista.

Ainda pela Tabela I percebemos que o modelo composto $y^{*}$ obtém uma diminuição de cerca de $79 \%$ para o índice NRMSE e de $22 \%$ para a energia da autocorrelação do vetor de resíduos de predição livre se comparados com os modelos $y_{1}$ e $y_{2}$ utilizados em sua obtenção.

\section{CONCLUSÕES}

Este artigo aplicou o método de combinação paralela de modelos apresentado em Rede Combinatória de Modelos Não Lineares - Parte I: Método e Aplicação em Identificação Multiobjetivo no contexto da computação aritmética. Um modelo foi obtido pela combinação paralela de dois submodelos pertencentes a um conjunto de representações obtidas a partir do Mínimos Quadrados Intervalar. Uma formulação matemática para o cálculo dos pesos foi apresentada e os resultados experimentais mostraram uma melhora no índice NRMSE e na autocorrelação do vetor de resíduos, se comparado com o procedimento clássico de identificação ou com o modelo do ponto médio do procedimento de identificação intervalar. O modelo composto obteve também um melhor desempenho comparado com os modelos utilizados em sua obtenção.

Como trabalho futuro pretende-se utilizar a abordagem apresentada no contexto da extensão da precisão de sistemas de aquisição. Assim modelos intervalares podem ser obtidos a partir de sistemas de aquisição com limitações em precisão e um único modelo pode ser interpolado a partir de uma amostra considerada precisa do sistema, 

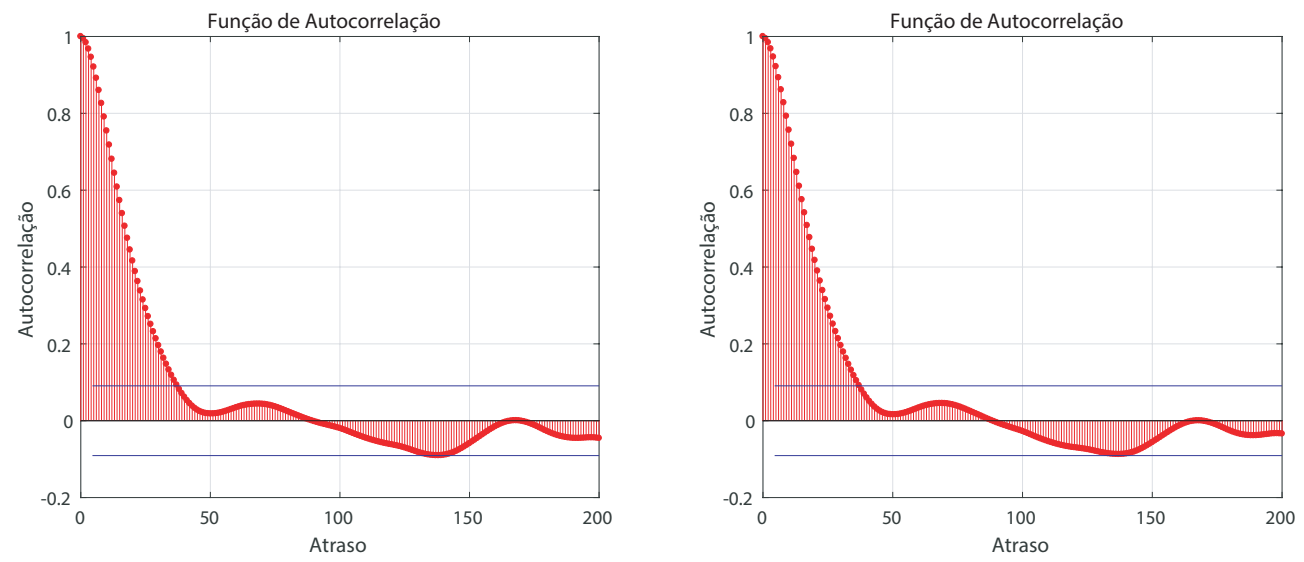

Figura 3. Autocorrelação do vetor de resíduos $\xi$ para o modelo combinado à esquerda e para o modelo identificado por um procedimento padrão de identificação à direita.
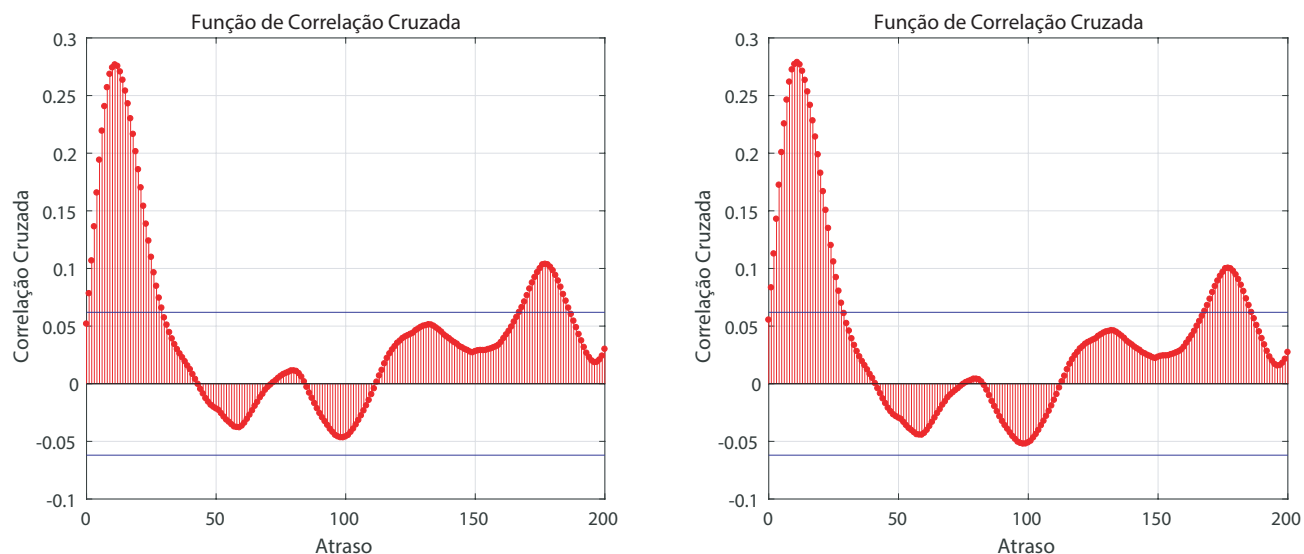

Figura 4. Correlação cruzada entre o vetor de resíduos $\xi$ e o vetor de entrada $u$ para o modelo composto à esquerda $\mathrm{e}$ para o modelo identificado por um procedimento padrão de identificação à direita.

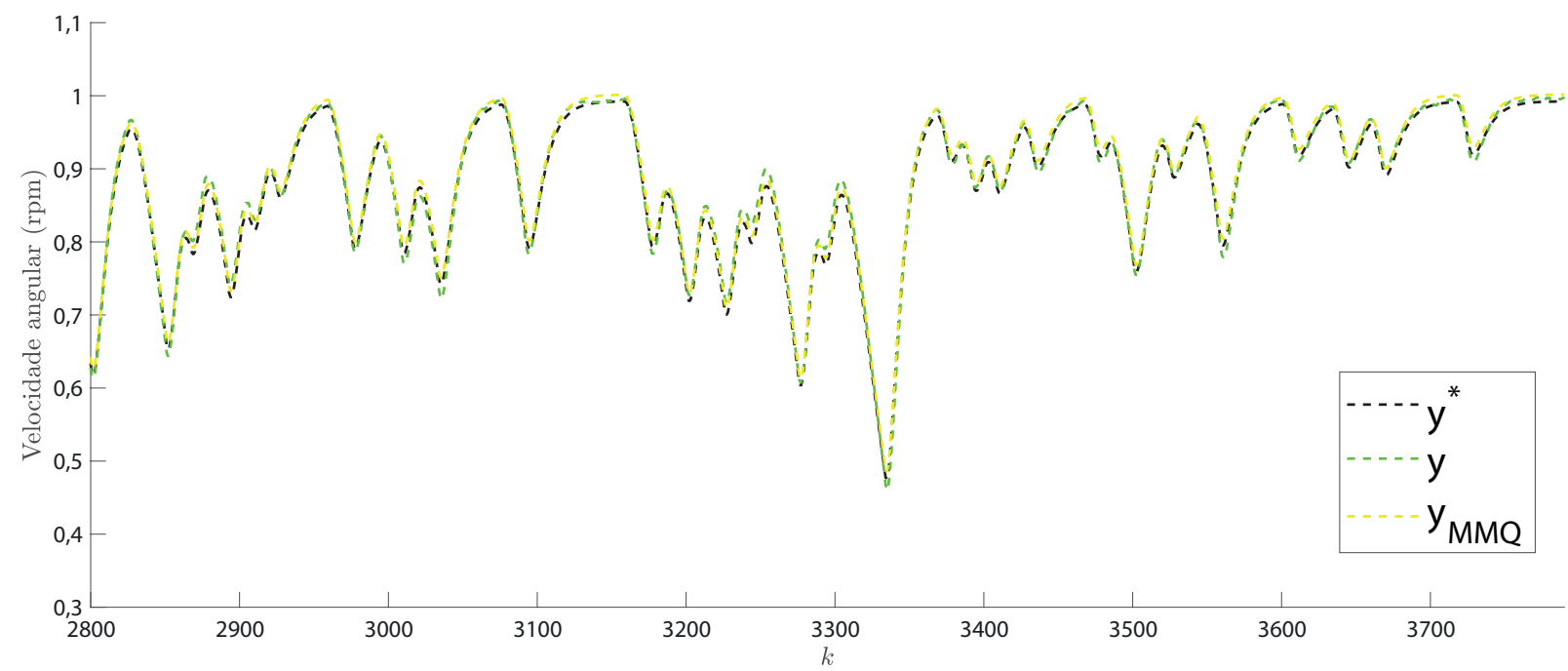

Figura 5. Comparação entre resposta do modelo composto, resposta real do sistema e resposta obtida por procedimento padrão de identificação

traduzindo assim modelos identificados em um ambiente de baixa precisão em um modelo em um ambiente de alta precisão.

\section{AGRADECIMENTOS}

O presente trabalho foi realizado com apoio da Coordenação de Aperfeiçoamento de Pessoal de Nível Superior - Bra- 


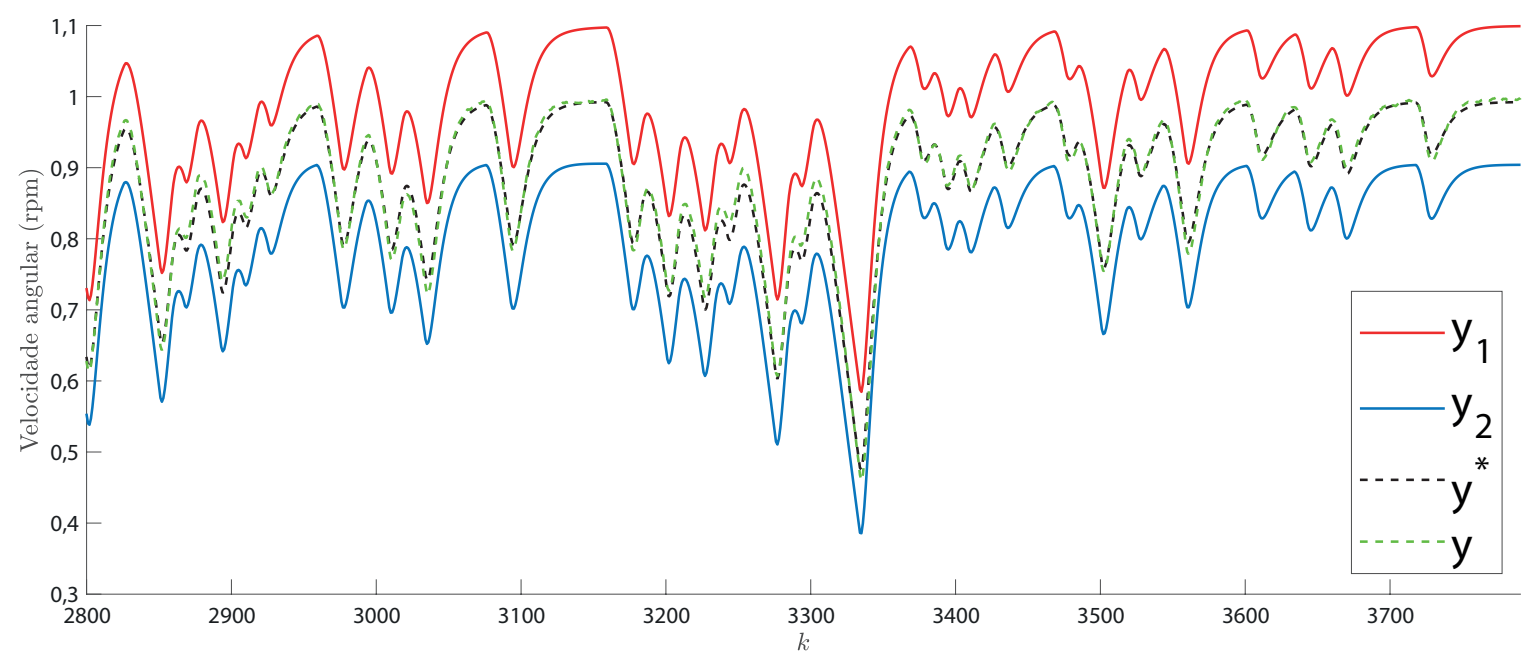

Figura 6. Resposta dos submodelos utilizados na interpolação, do modelo interpolado e resposta real do sistema

sil (CAPES) - Código de Financiamento 001. Os autores também agradecem à FAPEMIG, CNPq, e à Universidade Federal de São João del Rei pelo apoio.

\section{REFERÊNCIAS}

Aguirre, L. (2015). Introdução à Identificação de Sistemas - Técnicas Lineares e Não-Lineares Aplicadas a Sistemas Reais. Editora UFMG.

Aguirre, L.A. and Billings, S.A. (1995). Improved structure selection for nonlinear models based on term clustering. International Journal of Control, 62(3), 569-587.

Aguirre, L.A., Rodrigues, G.G., and F., J.C.R. (1998). Identificação de sistemas não-lineares utilizando modelos NARMAX polinomiais - uma revisão e novos resultados. SBA Controle \& Automação, 9(2), 90-106.

Aguirre, N., Ikhouane., F., Rodellar, J., and Christenson3, R. (2011). Parametric identification of the Dahl model for large scale MR dampers. Structural Control and Health Monitoring, 19(1), 88-106.

Boda, Y., Vignoni, A., G.Reynoso-Meza, and J.picó (2016). Parameter identification in synthetic biological circuits using multi-objective optimization. IFACPapersOnLine.

Chen, S. and Billings, S.A. (1989). Representations of nonlinear systems: The NARMAX model. International Journal of Control, 49(3), 1013-1032.

Chen, S. and Billings, S.A. (1992). Neural networks for nonlinear dynamic system modelling and identification. International Journal of Control.

Chevtchenko, S.F., Vale, R.F., and Macario, V. (2018). Multi-objective optimization for hand posture recognition. Expert Systems with Applications, 92, 170-181.

Gao, J., He, Q., Gao, H., Zhan, Z., and Wu, Z. (2018). Design of an efficient multi-objective recognition approach for 8-ball billiards vision system. Kuwait Journal of Science, 45(1), 39-53.

Hammel, S.M., Yorke, J.A., and Grebogi, C. (1987). Do numerical orbits of chaotic dynamical processes represent true orbits. Journal of Complexity, 3(2), 136-145.

Leontaritis, I.J. and Billings, S.A. (1985a). Input-output parametric models for non-linear systems Part I: De- terministic non-linear systems. International Journal of Control, 41(2), 303-328.

Leontaritis, I.J. and Billings, S.A. (1985b). Input-output parametric models for nonlinear systems, part II: stochastic nonlinear system. International Journal of Control, 41(4), 329-344.

Martins, S.A.M., Barbosa, A.M., and Nepomuceno, E.G. (2013). Determinação do Número ed Termos de Modelos Polonomiais: O critério de Informação de Akaike Multiobjetivo. Simpósio Brasileiro de Automação Inteligente.

Milovanović, M.B., Antić, D.S., and Rajić, M.N. (2017). Wood resource management using an endocrine NARX neural network. European Journal of Wood and Wood Products.

Moore, R.E., Kearfott, R.B., and Cloud, M.J. (2009). Introduction to Interval Analysis.

Narendra, K. and Parthasarathy, K. (1990). Identification and control of dynamical systems using neural networks. IEEE Transactions on Neural Networks, 1(1), 4-27.

Nepomuceno, E.G. and Martins, S.A. (2016). A lower bound error for free-run simulation of the polynomial NARMAX. Systems Science and Control Engineering, $4(1), 50-58$.

Nepomuceno, E.G., Martins, S.A., Silva, B.C., Amaral, G.F., and Perc, M. (2018). Detecting unreliable computer simulations of recursive functions with interval extensions. Applied Mathematics and Computation, 329, 408-419.

Nepomuceno, E.G. (2014). Convergence of recursive functions on computers. The Journal of Engineering, 327(5), $2-4$.

Ok, S.y., Jung, S., and Song, J. (2018). Multiobjective Optimization Approach for Robust Bridge Damage Identification against Sensor Noise. 2018.

Peixoto, M.L.C., Matos, M.T.R., Júnior, W.R.L., Martins, S.A., and Nepomuceno, E.G. (2017). Identificação de Sistemas Dinâmicos com Aritmética Intervalar. Simpósio Brasileiro de Automação Inteligente, 471-476.

Zhao, X., Wu, S., and Pan, H. (2018). A hybrid model of magnetorheological dampers based on generalized hysteretic biviscous operators. Journal of Intelligent Material Systems and Structures, 29(14), 2979-2985. 